\section{(6) OPEN ACCESS}

\title{
A randomised, double-blind, parallel-group study of the safety and efficacy of subcutaneous tocilizumab versus intravenous tocilizumab in combination with traditional disease-modifying antirheumatic drugs in patients with moderate to severe rheumatoid arthritis (SUMMACTA study)
}

\author{
Gerd R Burmester, ${ }_{1}^{1}$ Andrea Rubbert-Roth, ${ }^{2}$ Alain Cantagrel, ${ }_{1}^{3}$ Stephen Hall, ${ }_{1}^{4}$ \\ Piotr Leszczynski, ${ }^{5}$ Daniel Feldman, ${ }^{6}$ Madura J Rangaraj, ${ }_{1}^{7}$ Georgia Roane, ${ }^{8}$ \\ Charles Ludivico, ${ }^{9}$ Peng Lu, ${ }^{10}$ Lucy Rowell, ${ }^{11}$ Min Bao, ${ }^{12}$ Eduardo F Mysler ${ }^{13}$
}

\begin{abstract}
Handling editor Tore K Kvien
- Additional material is published online only. To view please visit the journal online (http://dx.doi.org/10.1136/ annrheumdis-2013-203523).

For numbered affiliations see end of article.

\section{Correspondence to} Dr Gerd R Burmester, Department of Rheumatology and Clinical Immunology, Charité-Universitätsmedizin Berlin, Charitéplatz 1 , Berlin 10117, Germany; gerd.burmester@charite.de
\end{abstract}

Received 25 February 2013 Revised 13 June 2013 Accepted 9 July 2013 Published Online First 31 July 2013

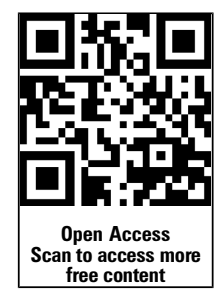

To cite: Burmester GR Rubbert-Roth $A$,

\section{ABSTRACT}

Objectives This study compared the efficacy and safety of subcutaneous (SC) versus intravenous (IV) formulations of tocilizumab in patients with rheumatoid arthritis with an inadequate response to diseasemodifying antirheumatic drugs (DMARD).

Methods Patients $(n=1262)$ were randomly assigned to receive tocilizumab-SC $162 \mathrm{mg}$ weekly+placebo-IV every 4 weeks or tocilizumab-IV $8 \mathrm{mg} / \mathrm{kg}$ every 4 weeks+placebo-SC weekly in combination with traditional DMARD. The primary outcome was to demonstrate the non-inferiority of tocilizumab-SC to tocilizumab-IV with regard to the proportion of patients in each group achieving an American College of Rheumatology (ACR) 20 response at week 24 using a $12 \%$ non-inferiority margin (NIM). Secondary outcomes were disease activity score using 28 joints (DAS28), ACR responses, health assessment questionnaire scores and safety assessments.

Results At week 24, 69.4\% (95\% Cl 65.5 to 73.2) of tocilizumab-SC-treated patients versus $73.4 \%(95 \% \mathrm{Cl}$ 69.6 to 77.1) of tocilizumab-IV-treated patients achieved an ACR20 response (weighted difference between groups $-4.0 \%, 95 \% \mathrm{Cl}-9.2$ to 1.2$)$; the $12 \%$ NIM was met. ACR50/70 responses, DAS28 and physical function improvements were comparable between the tocilizumabSC and tocilizumab-IV groups. The safety profiles of tocilizumab-SC and tocilizumab-IV were similar, and the most common adverse event was infection. Injection-site reactions (ISR) occurred more frequently in the tocilizumabSC group than in the tocilizumab-IV (placebo-SC) group. No anaphylaxis was reported over the 24 weeks. Conclusions Tocilizumab-SC 162 mg weekly demonstrated comparable efficacy to tocilizumab-IV $8 \mathrm{mg} / \mathrm{kg}$. The safety profile of tocilizumab-SC is consistent with the known and well-established safety profile of tocilizumab-IV, with the exception of a higher incidence of ISR, which were more common with tocilizumab-SC administration.

\section{INTRODUCTION}

Rheumatoid arthritis (RA) is a chronic, progressive, systemic autoimmune disease associated with joint inflammation. Advances in RA treatments have been made through the introduction of biological therapies, including anti-tumour necrosis factor (TNF) inhibitors, interleukin (IL)-1 receptor and IL-6 receptor (IL-6R) antagonists, an anti-CD20 agent and a T-cell co-stimulation modulator. ${ }^{1}$ Although these treatment options reduce disease activity, none are effective in all patients. While a patient's disease status and overall health should be central when choosing a therapy, differences in the route of administration and safety profiles of the agent can also affect the probability of a favourable response. ${ }^{1}$

Tocilizumab is a recombinant humanised anti-IL6R monoclonal antibody that blocks IL-6 from binding to the soluble and membrane-bound IL-6R and was initially developed as an intravenous (IV) infusion. The efficacy and safety of tocilizumab-IV were previously demonstrated as monotherapy and in combination with disease-modifying antirheumatic drugs (DMARD) in adult patients with RA in five phase 3 clinical trials. ${ }^{2}$ Tocilizumab is currently approved as an IV formulation for the treatment of RA, including in the USA and Europe.

A subcutaneous (SC) formulation of tocilizumab would offer patients an additional option that may allow self-administration. The tocilizumab-SC dose was selected based on pharmacokinetic/pharmacodynamic and limited efficacy and safety data from phase $1 / 2$ studies (see supplementary figure $\mathrm{S} 1$, available online only). ${ }^{3}$ To characterise further the efficacy and safety of tocilizumab-SC, the SUMMACTA study compared tocilizumab-SC $162 \mathrm{mg}$ weekly versus tocilizumab-IV $8 \mathrm{mg} / \mathrm{kg}$ every 4 weeks in adult patients with RA who have had an inadequate response to one or more DMARD.

\section{PATIENTS AND METHODS}

\section{Participants}

Patients ( $\geq 18$ years of age) with RA ( $\geq 6$ months, revised 1987 American College of Rheumatology (ACR) criteria) who met the following major criteria were included: swollen joint count of 4 or greater (66-joint count) and tender joint count of 4 or greater (68-joint count) at screening and baseline, 
C-reactive protein (CRP) $10 \mathrm{mg} / \mathrm{L}$ or greater and/or erythrocyte sedimentation rate (ESR) $28 \mathrm{~mm} / \mathrm{h}$ or greater at screening. Patients must have received one or more traditional DMARD at a stable dose for 8 weeks or longer before baseline. Patients were required to have had an inadequate response to DMARD (up to $20 \%$ of patients may have failed one or more anti-TNF). Before random assignment, patients discontinued all biological DMARD, including etanercept for 2 weeks or longer and infliximab, certolizumab, golimumab or adalimumab for 8 weeks or longer. Concomitant oral glucocorticoids $(\leq 10 \mathrm{mg} /$ day prednisone or equivalent) and non-steroidal anti-inflammatory drugs (up to the maximum recommended dose) were permitted if patients were on a stable dose for 4 weeks or longer before baseline.

Major exclusion criteria included ongoing rheumatic or inflammatory joint diseases other than RA, any active infections, history of malignancy, positive hepatitis B surface antigen or hepatitis $\mathrm{C}$ antibody, serious allergies to biological agents, previous treatment with tocilizumab, alkylating agents or celldepleting therapies or treatment with any investigational agent at less than 4 weeks of screening, and intra-articular or parenteral glucocorticoids or immunisation with a live/attenuated vaccine less than 4 weeks before baseline. Tuberculosis screening was managed according to local practice.

\section{Study design}

SUMMACTA was a 2-year, randomised, double-dummy, activecontrolled, parallel-group, phase 3 multicentre trial with a doubleblind period of 24 weeks followed by an open-label period of 72 weeks (results presented separately). Patients were stratified by geographical region and body weight category $(<60, \geq 60$ to $<100$ or $\geq 100 \mathrm{~kg}$ ). During the double-blind period, patients were randomly assigned $1: 1$ to receive $162 \mathrm{mg}$ of tocilizumab-SC $162 \mathrm{mg}$ per week+placebo-IV every 4 weeks or tocilizumab-IV $8 \mathrm{mg} / \mathrm{kg}$ every 4 weeks + placebo-SC weekly for 24 weeks (see supplement, available online only). Tocilizumab-SC/placebo injections were administered by prefilled syringe. After the first four treatments, subcutaneous injections could be administered at home by patients or caregivers; home injection information was recorded on diary cards. Dose modification to subcutaneously every 2 weeks or intravenously $4 \mathrm{mg} / \mathrm{kg}$ every 4 weeks or dose interruption was permitted for safety concerns.

\section{Outcomes and assessments}

The primary outcome was to evaluate the non-inferiority of tocilizumab-SC $162 \mathrm{mg}$ weekly to tocilizumab-IV $8 \mathrm{mg} / \mathrm{kg}$ regarding the proportion of patients in each group who achieved an ACR20 response at week 24 . Secondary endpoints included the proportion of patients who achieved an ACR50/70 response, remission based on the disease activity score using 28 joints (DAS28 <2.6) and a decrease from baseline of 0.3 or greater in the health assessment questionnaire-disability index (HAQ-DI) at week 24. Subgroup analyses for ACR responses and DAS28 remission were conducted across the three weight categories. Due to the non-inferiority design, the per-protocol (PP) population was used for the primary, secondary and subgroup analyses. The PP population was a subset of the intent-to-treat (ITT) population and excluded patients with major protocol violations that could potentially affect efficacy outcomes. All patients signed informed consent documents, and the study was conducted in accordance with the Declaration of Helsinki and good clinical practice.

\section{Safety}

The safety population included patients who received one or more dose of tocilizumab and had one or more post-dose safety assessment. Safety assessments included adverse events (AE), laboratory assessments, physical examination and vital signs. Laboratory monitoring was conducted at weeks 2 and 4 and every 4 weeks thereafter.

\section{Pharmacokinetics and pharmacodynamics}

Patients who provided one or more evaluable pharmacokinetic sample were included in the pharmacokinetic population. In the main study, the observed pre-dose tocilizumab concentration $\left(\mathrm{C}_{\text {trough }}\right)$ over 24 weeks was assessed; in the pharmacokinetic substudy at week 20, the mean area under the curve (AUC), maximum plasma concentration $\left(\mathrm{C}_{\max }\right)$ and $\mathrm{C}_{\text {trough }}$. pharmacodynamic parameters included CRP and ESR, were assessed as analysed in the safety population.

\section{Immunogenicity}

Blood samples were taken at baseline and throughout the study for anti-drug antibody assessment. Positive samples from the initial screening assay were analysed by a confirmation assay for specificity. The assays were performed as previously described using a bridging ELISA. ${ }^{4}$ If the confirmation assay was positive, an inhibition ELISA was performed to evaluate the neutralising potential of the anti-tocilizumab antibody.

\section{Statistical methods}

The time point for the primary analysis of the ACR20 response was week 24. To claim non-inferiority, the lower bound of the 95\% CI for the difference in ACR responses (tocilizumab-SC $162 \mathrm{mg}$ weekly minus tocilizumab-IV $8 \mathrm{mg} / \mathrm{kg}$ ) had to be greater than $-12 \%$. A non-inferiority margin of $12 \%$ was defined based on the results observed in the tocilizumab-IV trials. ${ }^{5-7}$

For the primary analysis, the $95 \%$ CI of the weighted difference in ACR responses between the groups at week 24 was calculated using Cochran-Mantel-Haenszel analysis. This difference was adjusted for the stratification factors of region and body weight. For all secondary endpoints, the differences in treatment effects and 95\% CI were reported. Sample size determination is outlined in the supplement (available online only).

\section{RESULTS}

\section{Patient disposition and baseline characteristics}

Of the 1262 patients randomly assigned, 631 received tocilizumab-SC $162 \mathrm{mg}$ weekly+placebo-IV and 631 received tocilizumab-IV $8 \mathrm{mg} / \mathrm{kg}+$ placebo-SC (figure 1 ). The PP population comprised 1095 patients $(n=558$, tocilizumab-SC $162 \mathrm{mg}$ weekly; $\mathrm{n}=537$, tocilizumab-IV $8 \mathrm{mg} / \mathrm{kg}$ ). The most common protocol violation leading to PP exclusion was a non-stable dose of DMARD for both groups.

Patient baseline demographics and clinical characteristics were balanced across the tocilizumab-SC and tocilizumab-IV groups in the PP (table 1) and safety populations (data not shown). The mean RA duration, tender joint counts, swollen joint counts and DAS28 scores were comparable between groups.

The prevalence of previous and current medications for RA was similar between groups. The proportion of patients who had inadequate responses to anti-TNF inhibitors was $22.5 \%$ in the tocilizumab-SC group and $21.6 \%$ in the tocilizumab-IV group; $79.7 \%$ of the tocilizumab-SC group and $81.5 \%$ of the tocilizumab-IV group received methotrexate.

\section{Efficacy}

The study met its primary endpoint by demonstrating the noninferiority of tocilizumab-SC $162 \mathrm{mg}$ weekly to tocilizumab-IV $8 \mathrm{mg} / \mathrm{kg}$. The proportion of tocilizumab-SC patients achieving 


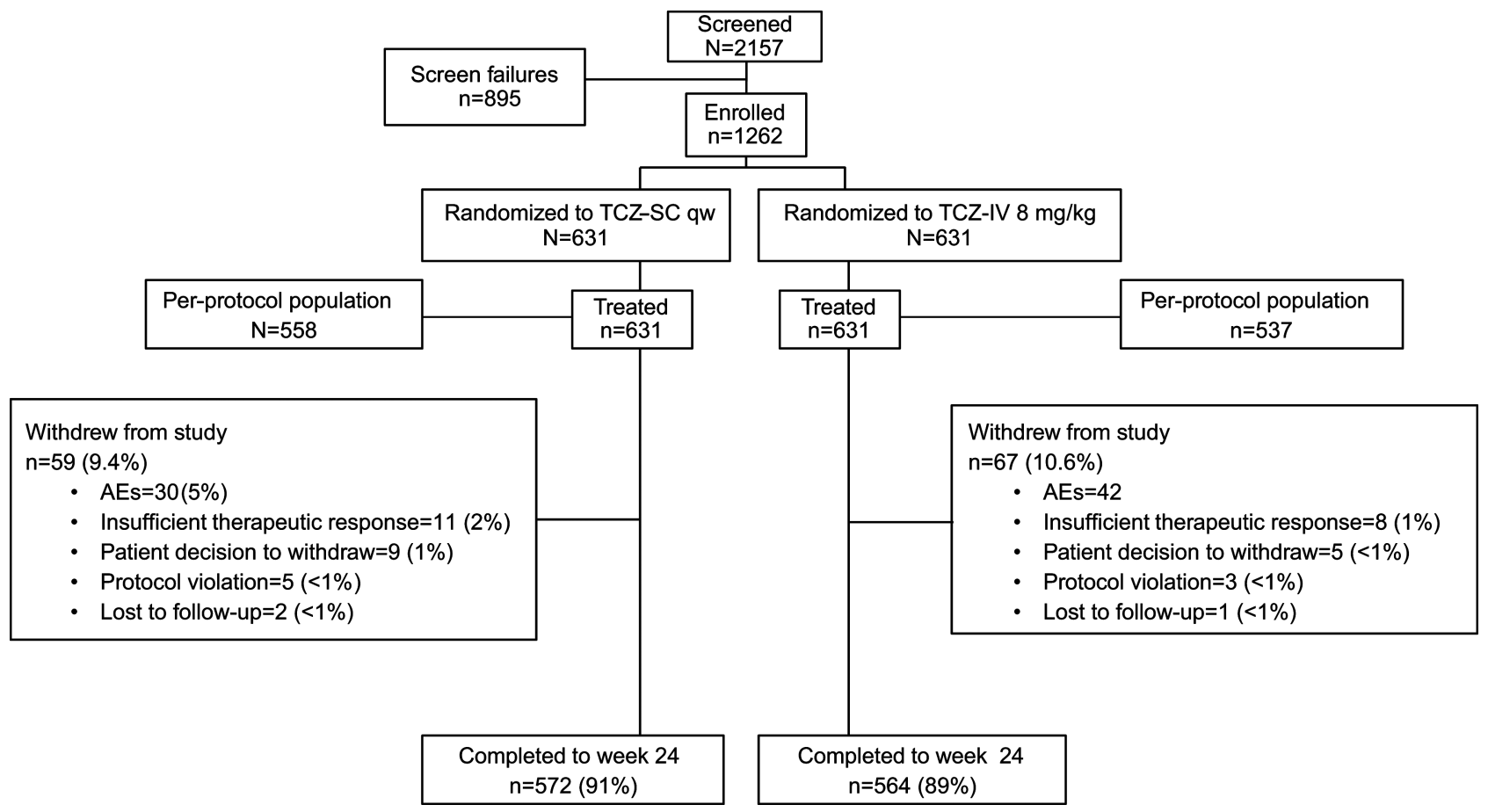

Figure 1 Patient disposition over 24 weeks. TCZ-IV, intravenous tocilizumab; TCZ-SC, subcutaneous tocilizumab. AE, adverse events; qw, weekly.

an ACR20 response at week 24 was $69.4 \%$ (95\% CI 65.5 to 73.2); for tocilizumab-IV patients, it was 73.4\% (95\% CI 69.6 to 77.1 ; figure $2 \mathrm{~A}$ ). The difference between groups was $-4.0 \%$ $(95 \%$ CI -9.2 to 1.2$)$, confirming the non-inferiority of tocilizumab-SC to tocilizumab-IV. The robustness of the primary endpoint analysis was supported by analysis in the ITT population: $-2.7 \%$ (95\% CI -7.6 to 2.2 ; see supplementary figure S2, available online only).

ACR20, ACR50 and ACR70 response rates over 24 weeks were similar between groups (figure $2 \mathrm{~A}$ ). The weighted differences in the proportion of ACR50 and ACR70 responders at week 24 were $-1.8 \%$ (95\% CI -7.5 to 4.0$)$ and $-3.8 \%(95 \%$ CI -9.0 to 1.3$)$, respectively.

The proportion of patients who achieved DAS28 remission over 24 weeks was comparable between groups (figure $2 \mathrm{~B}$ ). The weighted difference in the proportion of patients achieving DAS28 remission at week 24 was $0.9 \%$ (95\% CI -5.0 to 6.8 ). In post-hoc analyses, the proportion of patients who achieved simple disease activity index, clinical disease activity index and Boolean remission were also similar between groups (see supplementary figure S3, available online only).

The proportion of patients who achieved a decrease of 0.3 or greater in HAQ-DI score from baseline was similar between groups (figure 2C). The weighted difference in the proportion of patients achieving a decrease of 0.3 or greater in HAQ-DI score from baseline was $-2.3 \%$ (95\% CI -8.1 to 3.4$)$.

The proportion of patients achieving an ACR20/50/70 response (figure $3 \mathrm{~A}$ ) or DAS remission (figure $3 \mathrm{~B}$ ) was similar between the groups across the three body weights. The response in the heaviest weight category $(\geq 100 \mathrm{~kg})$ of both arms was lower and had more variation, which may be due to the lower number of patients in this weight category.

\section{Pharmacokinetics and pharmacodynamics}

The pharmacokinetic profiles of tocilizumab following subcutaneous and intravenous dosing varied due to the different routes of administration. Serum trough tocilizumab levels increased more rapidly in the tocilizumab-SC group than in the tocilizumab-IV group (see supplementary figure S4, available online only). With subsequent dosing, the trough tocilizumab levels plateaued by week 12 in both groups. The observed steady-state $C_{\text {trough }}( \pm S D)$ at week 24 was $42( \pm 27.4) \mu \mathrm{g} / \mathrm{mL}$ following tocilizumab-SC dosing and $18( \pm 14.2) \mu \mathrm{g} / \mathrm{mL}$ following tocilizumab-IV dosing.

Despite higher trough tocilizumab levels for tocilizumab-SC, the mean AUC and $\mathrm{C}_{\max }$ of tocilizumab at steady state were higher for tocilizumab-IV (see supplementary figure S5, available online only). Thirty-four patients participated in a pharmacokinetic substudy with more frequent sampling at week 20; 26 patients had sufficient data for analysis (tocilizumab-SC, $n=13$; tocilizumab-IV, $\mathrm{n}=13$ ) (see supplementary figure $\mathrm{S} 5$, available online only). At steady state from weeks 20 to 24 , the mean AUC $_{20-24 w k}$ was $30168 \mu \mathrm{g} \mathrm{h} / \mathrm{mL}$ for tocilizumab-SC and $41304 \mu \mathrm{g} \mathrm{h} / \mathrm{mL}$ for tocilizumab-IV. The mean $\mathrm{C}_{\max }$ values $( \pm S D)$ at week 20 were $52.7( \pm 27.3) \mu \mathrm{g} / \mathrm{mL}$ for tocilizumab-SC and $233( \pm 117) \mu \mathrm{g} / \mathrm{mL}$ for tocilizumab-IV.

CRP levels decreased in both groups after the first dose of tocilizumab (see supplementary figure S6, available online only). Thereafter, in both groups, CRP remained below the upper limit of normal (ULN; $0.99 \mathrm{mg} / \mathrm{dL}$ ) to week 24. The time course of CRP for tocilizumab-SC was comparable to that for tocilizumab-IV, although there was a trend towards slightly lower CRP levels in the tocilizumab-SC group. Similar results were observed for ESR (see supplementary figure S7, available online only).

\section{Safety}

The safety profile was similar between groups (safety population), except for more injection-site reactions (ISR) in the tocilizumab-SC group (table 2). Rates of AE, serious adverse events (SAE) and discontinuation due to $\mathrm{AE}$ were similar between groups. The proportion of patients in each group with a dose modification or interruption due to an $\mathrm{AE}$ was comparable (see supplementary table S1, available online only). The frequency of infection was $36.0 \%$ in the tocilizumab-SC group and 
Table 1 Baseline demographics (per-protocol population)

\begin{tabular}{|c|c|c|}
\hline & $\begin{array}{l}\text { Tocilizumab-SC } \\
162 \mathrm{mg} \\
\text { qw }(\mathrm{n}=558)\end{array}$ & $\begin{array}{l}\text { Tocilizumab-IV } \\
8 \mathrm{mg} / \mathrm{kg} \\
\text { q4w (n=537) }\end{array}$ \\
\hline \multicolumn{3}{|l|}{ Sex, n (\%) } \\
\hline Female & $461(82.6)$ & $444(82.7)$ \\
\hline Age, years, mean (SD) & $52.4(12.29)$ & $52.5(12.50)$ \\
\hline Weight, kg, mean (SD) & 74.07 (18.73) & $73.82(18.99)$ \\
\hline \multicolumn{3}{|l|}{ Weight group, n (\%) } \\
\hline$<60 \mathrm{~kg}$ & $131(23.5)$ & $129(24.0)$ \\
\hline $60-100 \mathrm{~kg}$ & $374(67.0)$ & $358(66.7)$ \\
\hline$\geq 100 \mathrm{~kg}$ & $53(9.5)$ & $50(9.3)$ \\
\hline Duration of RA, years, mean (SD) & $8.7(8.18)$ & $8.7(7.94)$ \\
\hline Tender joints (68-joint count), mean (SD) & $27.5(15.54)$ & $28.8(16.42)$ \\
\hline Swollen joints (66-joint count), mean (SD) & $15.1(9.03)$ & $16.8(10.55)$ \\
\hline HAQ-DI score, mean (SD) & $1.6(0.62)$ & $1.7(0.65)$ \\
\hline Patient's assessment of pain, mean (SD) & $60.2(22.48)$ & $61.8(21.86)$ \\
\hline $\begin{array}{l}\text { Patient's global assessment of disease } \\
\text { activity, mean (SD) }\end{array}$ & $67.3(21.65)$ & $67.5(21.63)$ \\
\hline $\begin{array}{l}\text { Physician's global assessment of disease } \\
\text { activity, mean (SD) }\end{array}$ & $61.2(17.91)$ & $62.6(18.55)$ \\
\hline RF positive, $\mathrm{n}(\%)$ & $456(73.5)^{*}$ & $465(74.4) \dagger$ \\
\hline ACPA positive, $n(\%)$ & $434(72.2) \ddagger$ & $471(74.4) \S$ \\
\hline CRP level, mg/dL, mean (SD) & $2.1(2.22)$ & $2.2(2.25)$ \\
\hline DAS28, mean (SD) & $6.6(1.00)$ & $6.7(1.01)$ \\
\hline Patients receiving methotrexate, $\mathbf{n}$ (\%) & $503(79.7)^{\star *}$ & $514(81.5)^{* *}$ \\
\hline $\begin{array}{l}\text { Patients receiving glucocorticoids at } \\
\text { baseline, } n(\%)\end{array}$ & $300(53.8)$ & $290(54.0)$ \\
\hline Previously failed anti-TNF treatment, $\mathrm{n}(\%)$ & $121(21.7)$ & $112(20.9)$ \\
\hline \multicolumn{3}{|c|}{ 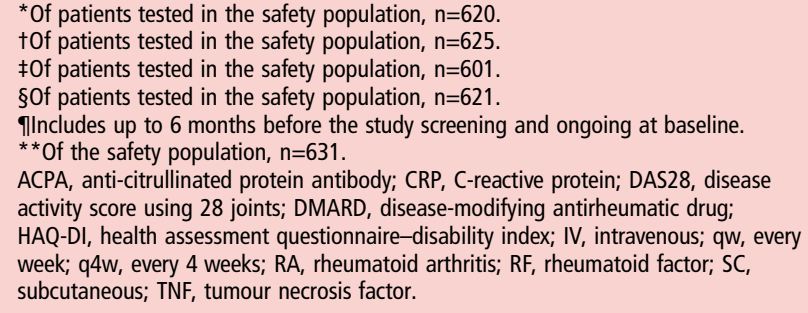 } \\
\hline
\end{tabular}

$39.1 \%$ in the tocilizumab-IV group. The most common AE was infection; among them, the most commonly reported preferred term was upper respiratory tract infection $(7.3 \%$ tocilizumab-SC and $11.6 \%$ tocilizumab-IV). The most common SAE was infection (1.4\% in both groups). Serious infections of pneumonia occurred in two patients in each group and bacterial arthritis occurred in two patients in the tocilizumab-IV group. The most common reason for withdrawal due to $\mathrm{AE}$ in both groups was infection (1.1\% tocilizumab-SC and $1.3 \%$ tocilizumab-IV). No obvious differences were observed between treatment groups for incidences of $\mathrm{AE}$ and SAE across the three weight categories (see supplementary table S2, available online only).

No deaths were reported for patients receiving tocilizumab-SC. One death was reported in the tocilizumab-IV group, attributable to sepsis due to bacterial arthritis.

Three SAE of malignancy $(<1 \%)$ occurred in the tocilizumab-SC group and one $(<1 \%)$ in the tocilizumab-IV group. In the tocilizumab-SC group, breast cancer was diagnosed in two patients and a brain neoplasm in one patient. In the tocilizumab-IV group, squamous cell carcinoma was diagnosed in one patient.

ISR were more common in the tocilizumab-SC group $(10.1 \%)$ than in the tocilizumab-IV group (2.4\%), in which patients also received placebo-SC. All ISR were non-serious and
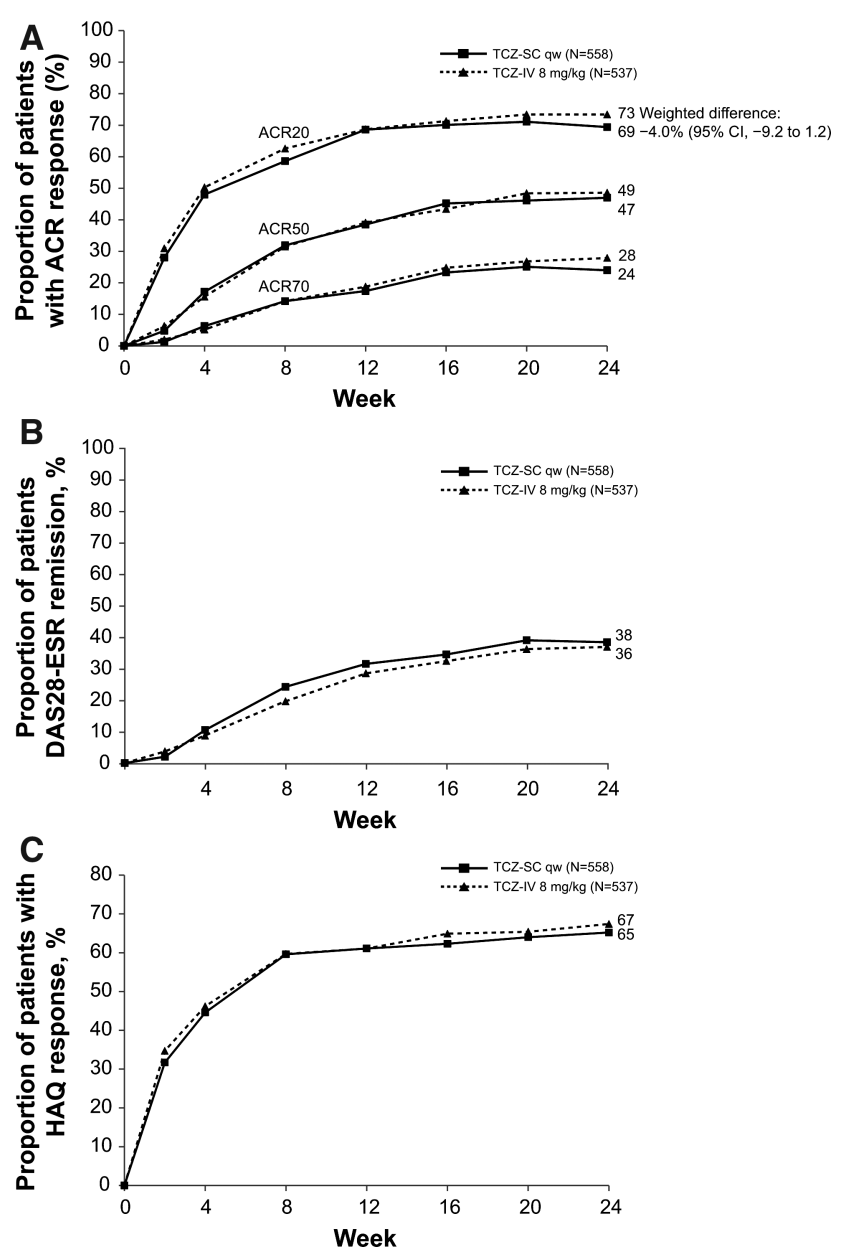

Figure 2 Disease activity and physical function over 24 weeks for patients in the per-protocol (PP) population. (A) Proportion of patients in the PP population treated with either subcutaneous tocilizumab (TCZ-SC; $n=558$ ) or intravenous tocilizumab (TCZ-IV; $n=537$ ) achieving $20 \%, 50 \%$ and $70 \%$ improvements per American College of Rheumatology criteria (ACR20, ACR50 and ACR70) over 24 weeks. (B) Proportion of patients achieving remission based on disease activity score using 28 joints (DAS28) based on erythrocyte sedimentation rate (ESR <2.6) over 24 weeks. (C) Proportion of patients achieving a health assessment questionnaire (HAQ) response (improvement of $\geq 0.3$ from baseline) over 24 weeks. qw, weekly.

common terminology criteria for adverse events grade 1 or 2 ; none required dose interruption or withdrawal.

No anaphylaxis events were reported. Five SAE were observed within $24 \mathrm{~h}$ of infusion or injection and evaluated as 'related' to study treatment; of these, three were medically consistent with hypersensitivity and led to early study withdrawal (two events in the tocilizumab-SC group and one event in the tocilizumab-IV group). All events resolved without sequelae.

After initiation of tocilizumab, the majority of shifts in ALT and AST from normal at baseline were threefold or less the ULN; ALT shifts occurred in more patients in the tocilizumab-SC group compared with the tocilizumab-IV group (46\% vs $39 \%$, see supplementary table S3, available online only). No differences between groups were observed for ALT and AST shifts from normal at baseline to a value between more than three times and five times or less ULN, or from normal to more than five times ULN. One patient in the tocilizumab-SC group and three patients in the tocilizumab-IV group had sustained consecutive ALT elevations (an elevation from the time of the first elevation to the last record). One patient in the 

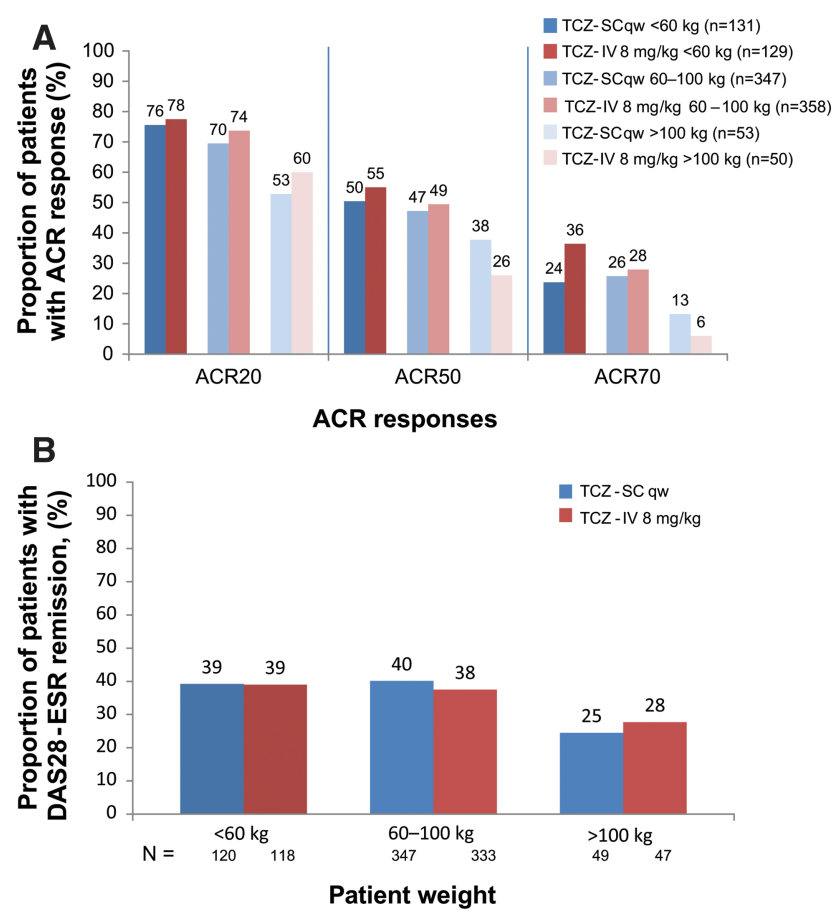

Figure 3 Proportion of patients stratified by weight in the per-protocol population treated with either subcutaneous tocilizumab (TCZ-SC; $n=558$ ) or intravenous tocilizumab (TCZ-IV; $n=537$ ) (A) achieving $20 \%, 50 \%$ and $70 \%$ improvements per American College of Rheumatology criteria (ACR20, ACR50 and ACR70) over 24 weeks and (B) achieving remission based on disease activity score using 28 joints (DAS28) based on erythrocyte sedimentation rate (ESR <2.6) over 24 weeks. qw, weekly.

tocilizumab-SC group and two patients in the tocilizumab-IV group had sustained consecutive AST elevations. Five patients $(0.8 \%)$ in the tocilizumab-SC group and seven patients $(1.1 \%)$ in the tocilizumab-IV group prematurely discontinued because of elevated liver transaminases. Among patients with a low neutrophil count after initiating treatment, most experienced common terminology criteria grade 1 or 2 neutropenia (see supplementary table S3, available online only). Grades 1 and 2 events of neutropenia were reported for a slightly higher proportion of patients in the tocilizumab-SC group compared with the tocilizumab-IV group. No differences were observed between groups for grades 3 or 4 neutropenia. One patient in the tocilizumab-SC group experienced a sustained consecutive neutrophil decline. For patients with a low platelet count, almost all events were grade 1 . The proportion of patients with an increase in total cholesterol from less than $200 \mathrm{mg} / \mathrm{dL}$ at baseline to more than $200 \mathrm{mg} / \mathrm{dL}$ at the last observation was slightly higher in the tocilizumab-SC group than in the tocilizumab-IV group (50\% vs $42 \%)$. The largest categorical shift in total cholesterol (from $<200$ to $\geq 240 \mathrm{mg} / \mathrm{dL}$ ) was more common in tocilizumab-SC patients than in tocilizumab-IV patients (7\% vs 4\%). Clinically relevant shifts in low-density lipoprotein cholesterol, high-density lipoprotein cholesterol and triglyceride levels were similar between groups.

\section{Immunogenicity}

Five patients in each group were positive $(0.8 \%$ and $0.8 \%)$ for both the confirmation and neutralising assays postbaseline. No patients with serious hypersensitivity events developed anti-tocilizumab antibodies. One patient in the tocilizumab-SC group with anti-tocilizumab antibodies had an

\begin{tabular}{|c|c|c|}
\hline & $\begin{array}{l}\text { Tocilizumab-SC } \\
162 \mathrm{mg} \mathrm{qw} \\
(\mathrm{n}=631) 289.82 \mathrm{PY}\end{array}$ & $\begin{array}{l}\text { Tocilizumab-IV } \\
8 \mathrm{mg} / \mathrm{kg} \mathrm{q4w} \\
(\mathrm{n}=631) 288.39 \mathrm{PY}\end{array}$ \\
\hline \multicolumn{3}{|l|}{$\mathrm{AE}$} \\
\hline Total AE, $n$ & 1747 & 1697 \\
\hline Patients with $\geq 1 \mathrm{AE}, \mathrm{n}(\%)$ & $481(76.2)$ & $486(77.0)$ \\
\hline $\begin{array}{l}\text { Discontinuation due to } A E \\
n(\%)\end{array}$ & $30(4.8)$ & $42(6.7)$ \\
\hline \multicolumn{3}{|l|}{ SAE } \\
\hline Total SAE, $\mathrm{n}$ & 34 & 43 \\
\hline Patients with $\geq 1 \mathrm{SAE}, \mathrm{n}(\%)$ & $29(4.6)$ & $33(5.2)$ \\
\hline SAE per 100 PY $(95 \% \mathrm{Cl})$ & 11.73 (8.12 to 16.39$)$ & 14.91 (10.79 to 20.08 ) \\
\hline \multicolumn{3}{|l|}{ SI } \\
\hline Total SI & 9 & 9 \\
\hline Patients with $\geq 1 \mathrm{SI}, \mathrm{n}(\%)$ & $9(1.4)$ & $9(1.4)$ \\
\hline SI per $100 \mathrm{PY}(95 \% \mathrm{Cl})$ & 3.11 (1.25 to 5.89$)$ & 3.47 (1.66 to 6.38$)$ \\
\hline $\begin{array}{l}\text { Serious hypersensitivity } \\
\text { reactions* }{ }^{*} \mathrm{n}(\%)\end{array}$ & $2(<1)$ & $3+(<1)$ \\
\hline \multicolumn{3}{|l|}{ ISR } \\
\hline Patients with ISR, n (\%) & $64(10.1)$ & $15(2.4)$ \\
\hline ISR, $\mathrm{n}$ & 168 & 94 \\
\hline Erythema, $\mathrm{n}(\%)$ & $28(4.4)$ & $5(0.8)$ \\
\hline Pain, n (\%) & $12(1.9)$ & $5(0.8)$ \\
\hline Pruritus, n (\%) & $14(2.2)$ & $0(0)$ \\
\hline Haematoma, n (\%) & $5(0.8)$ & $5(0.8)$ \\
\hline $\begin{array}{l}\text { Dose interruption or study } \\
\text { withdrawal because of ISR, } n\end{array}$ & 0 & 0 \\
\hline Death, $n(\%)$ & $0(0)$ & $1(<1)$ \\
\hline \multicolumn{3}{|c|}{$\begin{array}{l}\text { "Serious hypersensitivity was defined as an SAE occurring during or within } 24 \mathrm{~h} \text { of } \\
\text { the injection or infusion, excluding ISR, and evaluated as 'related' to study treatment } \\
\text { by the investigator. } \\
\text { tOf the three events in the tocilizumab-IV group, one was cellulitis and one was } \\
\text { retinal artery occlusion; these two events were not considered consistent with a } \\
\text { serious hypersensitivity reaction. } \\
\text { AE, adverse event; ISR, injection-site reaction; IV, intravenous; PY, patient-years; qw, } \\
\text { every week; q4w, every } 4 \text { weeks; SAE, serious adverse event; SC, subcutaneous; SI, } \\
\text { serious infection. }\end{array}$} \\
\hline
\end{tabular}

ISR. No patients with detected anti-tocilizumab antibodies withdrew because of an insufficient therapeutic response or loss of efficacy (defined as patients from the ITT population who withdrew due to insufficient therapeutic response or who experienced an ACR50 or DAS-ESR-based European League Against Rheumatism) good response before withdrawal). Based on the limited number of patients who developed antitocilizumab antibodies, no impact of antibodies on the pharmacokinetics of tocilizumab was observed.

\section{DISCUSSION}

Tocilizumab-IV is an efficacious therapy with an acceptable risk/ benefit profile for patients with RA. The SUMMACTA study analysed whether tocilizumab-SC $162 \mathrm{mg}$ weekly was non-inferior to tocilizumab-IV $8 \mathrm{mg} / \mathrm{kg}$ in patients with $\mathrm{RA}$ with an inadequate response to DMARD, which may have included one or more anti-TNF inhibitors. The primary endpoint was met by demonstrating that the ACR20 response at week 24 for patients treated with tocilizumab-SC was non-inferior to that of patients treated with tocilizumab-IV. The safety profiles of tocilizumab-SC and tocilizumab-IV were similar, except for a higher incidence of ISR more commonly observed with tocilizumab-SC administration.

The ACR20 response rate for the tocilizumab-IV patients in the PP population was higher than that observed in previous tocilizumab-IV studies. ${ }^{5-8}$ Baseline disease characteristics were comparable with previous tocilizumab-IV studies, so the higher 
ACR20 response rate in this study may reflect that all patients received active tocilizumab treatment, whereas previous tocilizumab-IV studies included a placebo group or other comparator during the double-blind phase.

The CRP time course was comparable between groups. There was a trend towards slightly lower CRP levels in the tocilizumab-SC group, which was consistent with the higher $\mathrm{C}_{\text {trough }}$ concentrations in the tocilizumab-SC group over 24 weeks. These results were similar to those of previous phase $1 / 2$ studies for tocilizumab-SC $162 \mathrm{mg}$ weekly, thus validating the assumptions made for the dose assessed.

The safety profiles between groups were similar in this study and consistent with previous studies of long-term administration of tocilizumab-IV, with infections being the most common $\mathrm{AE}$ and SAE $;^{5-8}$ consistent with other subcutaneous RA treatments, higher ISR rates were reported in tocilizumab-SC than in tocilizumab-IV. The incidence rate $(10.1 \%)$ of ISR in the tocilizumab-SC group was similar to that reported at 24 weeks in other studies in patients with RA who received subcutaneous anti-TNF inhibitors. 910

Comparison of the incidence of antibodies to tocilizumab-SC with other subcutaneous RA therapies is difficult because the measurement of antibody positivity (including neutralising antibodies) is highly dependent on assay sensitivity, specificity and methodology. In this study, the proportion of patients in both groups who developed anti-tocilizumab antibodies was low and comparable; no correlation was observed between antibody development and $\mathrm{AE}$ or clinical response. Therefore, the immunogenicity potential following tocilizumab-SC treatment was considered low based on available data.

A limitation of the data is that long-term efficacy and safety have not been explored. Longer observation from this study and additional data from other studies with tocilizumab-SC will provide further information related to immunogenicity and AE.

In summary, the non-inferiority of tocilizumab-SC $162 \mathrm{mg}$ weekly to tocilizumab-IV $8 \mathrm{mg} / \mathrm{kg}$ every 4 weeks was demonstrated. Tocilizumab-SC demonstrated efficacy and clinical safety profiles comparable with those of tocilizumab-IV, with the exception of a higher incidence of ISR more commonly seen with tocilizumab-SC. The tocilizumab-SC formulation could provide an additional, more convenient administration option and opportunity for home injection for patients with RA.

\footnotetext{
Author affiliations

${ }^{1}$ Charité-Universitätsmedizin Berlin, Free University and Humboldt University of Berlin, Berlin, Germany

${ }^{2}$ Klinikum der Universität zu Köln, Koln, Germany

${ }^{3}$ Centre Hospitalier Universitaire de Toulouse, Toulouse, France

${ }^{4}$ Cabrini Medical Centre, Malvern, Australia

5. Strus Poznan Municipal Hospital, Poznan University of Medical Sciences, Poland

${ }^{6}$ Universidade Federal de São Paulo, São Paulo, Brazil

${ }^{7}$ Arthritis \& Diabetes Clinic, Inc, Monroe, Louisiana, USA

${ }^{8}$ Rheumatology Associates, P.A., Charleston, South Carolina, USA

${ }^{9}$ East Penn Rheumatology Associates, PC, Bethlehem, Pennsylvania, USA

${ }^{10}$ Hoffmann-La Roche Inc, Nutley, New Jersey, USA

${ }^{11}$ Roche Products Limited, Welwyn Garden City, UK

${ }^{12}$ Genentech, Inc, South San Francisco, California, USA

${ }^{13}$ Organizacion Medica de Investigación, Buenos Aires, Argentina
}

Correction notice This article has been corrected since it was published Online First. Figure $2 \mathrm{~A}$ has been amended, and the following sentence amended to read: The most common AE was infection; among them, the most commonly reported preferred term was upper respiratory tract infection (7.3\% tocilizumab-SC and $11.6 \%$ tocilizumab-IV). The most common SAE was infection (1.4\% in both groups).

Acknowledgements The authors wish to thank all investigators and patients who participated in the study and all members of the WA22762 study team. Support for third-party writing assistance for this manuscript, furnished by Denise Kenski, PhD of Health Interactions, was provided by F. Hoffmann-La Roche, Ltd.
Contributors $L R$ and $M B$ designed the study, analysed and interpreted the data. PL analysed and interpreted the data. GRB, AR-R, AC, SH, PL, DF, MJR, GR, CL and EFM were involved in generating the data at their clinical research sites. All authors were involved in writing the manuscript and approved it.

Funding This study was funded by Roche. F. Hoffmann-La Roche, Ltd (Roche) sponsored the study, participated in the design of the study as well as in the collection, analysis and interpretation of the data. This manuscript was reviewed by Roche, but the decision to submit and publish this manuscript was contingent only on the approval of the lead author and co-authors, including those employed by Roche.

Competing interests GRB has received research grants from Roche, Abbott, Pfzier, UCB, Merck Sharp and Dohme and Bristol-Myers Squibb; received consulting fees from Roche, Chugai, Pfizer, UCB and Bristol-Myers Squibb; and was on the speaker bureaux for Roche, Pfizer, Merck Sharp and Dohme, Abbott and Bristol-Myers Squibb. $A R-R$ has received research grants from Roche and Pfizer; received consulting fees from Roche, Chugai, Pfizer, UCB and Merck Sharp and Dohme; and was on the speaker bureaux for Roche and UCB. AC has received research grants from UCB and Pfizer and received consulting fees from Roche, Chugai, Pfizer, UCB, Abbott and Bristol-Myers Squibb. PL has received consulting fees from Roche. MJR has received research grants from Roche. $\mathrm{CL}$ has received research grants from Roche, Bristol-Myers Squibb, Pfizer, Human Genome Science, Eli Lilly and Sanofi Aventis and was on the speaker bureau for BMS. EFM has received research grants and consulting fees from Roche and was on the speaker bureau for Roche. PL, LR and MB are employed by Roche. SH, DF and GR have no competing interests to declare.

\section{Patient consent Obtained.}

Ethics approval The study was conducted in accordance with the Declaration of Helsinki and good clinical practice.

Provenance and peer review Not commissioned; externally peer reviewed.

Open Access This is an Open Access article distributed in accordance with the Creative Commons Attribution Non Commercial (CC BY-NC 3.0) license, which permits others to distribute, remix, adapt, build upon this work non-commercially, and license their derivative works on different terms, provided the original work is properly cited and the use is non-commercial. See: http://creativecommons.org/licenses/by-nd/3.0/

\section{REFERENCES}

1 Scott DL. Biologics-based therapy for the treatment of rheumatoid arthritis. Clin Pharmacol Ther 2012;91:30-43.

2 Maini RN, Taylor PC, Szechinski J, et al. Double-blind randomized controlled clinical trial of the interleukin- 6 receptor antagonist, tocilizumab, in European patients with rheumatoid arthritis who had an incomplete response to methotrexate. Arthritis Rheum 2006;54:2817-29.

3 Ohta S, Tsuru T, Terao K. Optimal dose prediction by pharmacokinetic and biomarker response of subcutaneous tocilizumab treatment a Phase I/II study evaluating the safety, pharmacokinetics and clinical response in patients with rheumatoid arthritis. Arthritis Rheum 2010;62:S1115.

4 Stubenrauch $\mathrm{K}$, Wessels $U$, Birnboeck $H$, et al. Subset analysis of patients experiencing clinical events of a potentially immunogenic nature in the pivotal clinical trials of tocilizumab for rheumatoid arthritis: Evaluation of an antidrug antibody ELISA using clinical adverse event-driven immunogenicity testing. Clin Ther 2010;32:1597-609.

5 Smolen JS, Beaulieu A, Rubbert-Roth A, et al. Effect of interleukin-6 receptor inhibition with tocilizumab in patients with rheumatoid arthritis (OPTION study): a double-blind, placebo-controlled, randomised trial. Lancet 2008;371:987-97.

6 Genovese MC, MCKay JD, Nasonov EL, et al. Interleukin-6 receptor inhibition with tocilizumab reduces disease activity in rheumatoid arthritis with inadequate response to disease-modifying antirheumatic drugs: the tocilizumab in combination with traditional disease-modifying antirheumatic drug therapy study. Arthritis Rheum 2008;58:2968-80.

7 Kremer JM, Blanco R, Brzosko M, et al. Tocilizumab inhibits structural joint damage in rheumatoid arthritis patients with inadequate responses to methotrexate: results from the double-blind treatment phase of a randomized placebo-controlled trial of tocilizumab safety and prevention of structural joint damage at one year. Arthritis Rheum 2011;63:609-21.

8 Emery P, Keystone E, Tony HP, et al. IL-6 receptor inhibition with tocilizumab improves treatment outcomes in patients with rheumatoid arthritis refractory to anti-tumour necrosis factor biologicals: results from a 24-week multicentre randomised placebo-controlled trial. Ann Rheum Dis 2008;67:1516-23.

9 Keystone EC, Genovese MC, Klareskog L, et al. Golimumab, a human antibody to tumour necrosis factor \{alpha\} given by monthly subcutaneous injections, in active rheumatoid arthritis despite methotrexate therapy: the GO-FORWARD Study. Ann Rheum Dis 2009;68:789-96.

10 van de Putte LB, Atkins C, Malaise M, et al. Efficacy and safety of adalimumab as monotherapy in patients with rheumatoid arthritis for whom previous disease modifying antirheumatic drug treatment has failed. Ann Rheum Dis 2004:63:508-16. 